\title{
Decomposition of Timed Decision Tables and its Use in Presynthesis Optimizations
}

\author{
Jian Li \\ Department of Computer Science \\ University of Illinois, Urbana-Champaign \\ Urbana, Illinois 61801
}

\author{
Rajesh K. Gupta \\ Information \& Computer Science \\ University of California, Irvine \\ Irvine, California 92697
}

\begin{abstract}
Presynthesis optimizations transform a behavioral HDL description into an optimized $H D L$ description that results in improved synthesis results. In this paper we introduce the decomposition of Timed Decision Tables (TDT), a tabular model of system behavior. The TDT decomposition is based on the kernel extraction algorithm. By experimenting using named benchmarks, we demonstrate how TDT decomposition can be used in presynthesis optimizations.
\end{abstract}

\section{Introduction}

Presynthesis optimizations have been introduced in [1] as source-level transformations that produce "better" HDL descriptions. For instance, these transformations are used to reduce control-flow redundancies and make synthesis result relatively insensitive to the HDL codingstyle. They are also used to reduce resource requirements in the synthesized circuits by increasing component sharing at the behavior-level [2].

The TDT representation consists of a main table holding a set of rules (similar to the specification in a FSMD [3]), an auxiliary table which specifies concurrencies, data dependencies, and serialization relations among data-path computations, or actions, and a delay table which specifies the execution delay of each action.

The rule section of the model is based on the notions of condition and action. A condition may be the presence of an input, or the outcome of a test condition. A conjunction of several conditions defines a rule. A decision table is a collection of rules that map condition conjunctions into sets of actions. Actions include logic, arithmetic, input-output(IO), and message-passing operations. We associate an execution delay with each action. Actions are grouped into action sets, or compound actions. With each action set, we associate a

concurrency type of serial, parallel, or data-parallel [4]. The structure of the rule section can be found in [1]

In additional to the set of rules specified in a main table (the rule section), the TDT representation includes two auxiliary tables to hold additional information about the execution delay of each action, serialization, data dependency, and concurrency type between each pair of actions.

Example 1.1. Consider the following TDT:

\begin{tabular}{|c|c|c|c|c|c|c|c|}
\hline & $c_{1}$ & $\mathrm{Y}$ & $\mathrm{Y}$ & $\mathrm{N}$ & \multirow{2}{*}{\multicolumn{2}{|c|}{ delay }} & \\
\hline & $c_{2}$ & $Y$ & $\mathrm{~N}$ & $\mathrm{X}$ & & & \\
\hline & $a_{1,1}$ & 1 & 0 & 0 & & 1 & \\
\hline & $a_{1,2}$ & 1 & 0 & 0 & & 3 & \\
\hline & $a_{2,1}$ & 0 & 1 & 0 & & 4 & \\
\hline & $a_{2,2}$ & 0 & 1 & 0 & & 2 & \\
\hline & $a_{3,1}$ & 0 & 0 & 1 & & 6 & \\
\hline & $a_{3,2}$ & 0 & 0 & 1 & & 1 & \\
\hline & $a^{a_{1,1}}$ & $\begin{array}{l}a_{1,2} \\
\end{array}$ & $\overline{a_{2},}$ & & $a_{2,2}$ & $\begin{array}{l}a_{3,1} \\
\end{array}$ & $a_{3,2}$ \\
\hline$a_{1,1}$ & & $\mathrm{~s}$ & & & & & \\
\hline$a_{1,2}$ & & & & & & & \\
\hline$a_{2,1}$ & & & & & d & & \\
\hline$a_{2,2}$ & & & & & & & \\
\hline$a_{3,1}$ & & & & & & & $\mathrm{p}$ \\
\hline$\frac{a, 1}{a_{3,2}}$ & & & & & & & \\
\hline
\end{tabular}

An ' $\mathrm{X}$ ' in the condition column indicates a Don't Care associated with the particular condition. When $c_{1}=$ ' $\mathrm{Y}$ ' and $c_{2}=$ ' $\mathrm{Y}$ ', actions $a_{1,1}$ and $a_{1,2}$ are selected for execution. Since action $a_{1,2}$ is specified as a successor of $a_{1,1}$, action $a_{1,1}$ is executed with a one cycle delay followed by the execution of $a_{1,2}$. Symbols 'd' and 'p' indicate actions that are data-parallel and parallel actions respectively.

The execution of a TDT consists of two steps: (1) select a rule to apply, (2) execute the action sets that the selected rule maps to. More than two action sets may be selected for execution. The order in which to execute those action sets are determined by the concurrency types, serialization relations, and data dependencies specified among those action sets [4], indicated by 's', 'd', and ' $p$ ' in the table above. An action in a TDT may be another TDT. This is referred to as a call to the TDT contained as an action in the calling TDT.

Example 1.2. Consider the following calling hierarchy:

$T D T_{1}$

\begin{tabular}{|c||c|c|}
\hline$c_{1}$ & $\mathrm{Y}$ & $\mathrm{N}$ \\
\hline \hline$a_{1}$ & 1 & 0 \\
\hline$T D T_{2}$ & 0 & 1 \\
\hline
\end{tabular}

$T D T_{2}$

\begin{tabular}{|c||c|c|}
\hline$c_{2}$ & $\mathrm{Y}$ & $\mathrm{N}$ \\
\hline \hline$a_{2}$ & 1 & 0 \\
\hline$a_{3}$ & 0 & 1 \\
\hline
\end{tabular}


When $c_{1}=$ ' $\mathrm{N}$ ' the action needs to be invoked is the call to $T D T_{2}$, forces evaluation of condition $c_{2}$ resulting in actions $a_{2}$ or $a_{3}$ being executed. No additional information such as concurrency types needs to be specified between action $a_{1}$ and $T D T_{2}$ since they lie on different control paths.

Procedure/function calling hierarchy in input HDL descriptions results in a corresponding TDT hierarchy. TDTs in a calling hierarchy are typically merged to increase the scope of presynthesis optimizations. Merging flattens the calling hierarchy specified in original HDL descriptions. In this paper we present TDT decomposition which is the reverse of the merging process. By first flattening the calling hierarchy and then extracting the commonalities, we may find a more efficient behavior representation which leads to improved synthesis results. This allows us to restructure HDL code. In this paper, we introduce HDL code-restructuring using TDT merging and decomposition transformations. This optimization belongs to a group of presynthesis optimizations such as column/row reduction and action sharing that have been presented earlier $[1,4,2]$.

This paper is organized as follows. The next section introduces the notion of TDT decomposition and relates it to the problem of kernel extraction in an algebraic representation of TDTs. Section 3 presents an algorithm for TDT decomposition based on kernel extraction. Section 4 shows the implementation details of the algorithm and presents the experimental results. Finally, Section 5 concludes and presents our future plan.

\section{TDT Decomposition}

TDT decomposition is the process of replacing a flattened TDT with a hierarchical TDT that represents an equivalent behavior. As we mentioned earlier, decomposition is the reverse process of merging and together with merging, it allows us to produce HDL descriptions that are optimized for subsequent synthesis tasks and are relatively insensitive to coding styles. Since this decomposition uses procedure calling abstraction, arbitrary partitions of the table (condition/action) matrices are not useful.

Example 2.1. Consider the following TDT.

\begin{tabular}{|c||c|c|c|c|c|c|c|c|c|c|c|c|}
\hline$c_{1}$ & $\mathrm{Y}$ & $\mathrm{Y}$ & $\mathrm{Y}$ & $\mathrm{Y}$ & $\mathrm{Y}$ & $\mathrm{Y}$ & $\mathrm{Y}$ & $\mathrm{Y}$ & $\mathrm{N}$ & $\mathrm{N}$ & $\mathrm{N}$ & $\mathrm{N}$ \\
\hline$c_{2}$ & $\mathrm{Y}$ & $\mathrm{Y}$ & $\mathrm{Y}$ & $\mathrm{N}$ & $\mathrm{N}$ & $\mathrm{N}$ & $\mathrm{N}$ & $\mathrm{N}$ & & & & \\
\hline$c_{3}$ & & & & $\mathrm{Y}$ & $\mathrm{Y}$ & $\mathrm{Y}$ & $\mathrm{Y}$ & $\mathrm{N}$ & & & & \\
\hline$c_{4}$ & $\mathrm{Y}$ & $\mathrm{N}$ & $\mathrm{N}$ & & & & & & & & & \\
\hline$c_{5}$ & & $\mathrm{Y}$ & $\mathrm{N}$ & & & & & & & & & \\
\hline$c_{6}$ & & & & $\mathrm{Y}$ & $\mathrm{Y}$ & $\mathrm{N}$ & $\mathrm{N}$ & & $\mathrm{Y}$ & $\mathrm{Y}$ & $\mathrm{N}$ & $\mathrm{N}$ \\
\hline$c_{7}$ & & & & $\mathrm{Y}$ & $\mathrm{N}$ & $\mathrm{Y}$ & $\mathrm{N}$ & & $\mathrm{Y}$ & $\mathrm{N}$ & $\mathrm{Y}$ & $\mathrm{N}$ \\
\hline \hline$a_{1}$ & 1 & 1 & 1 & & & & & 1 & 1 & 1 & 1 & 1 \\
\hline$a_{2}$ & 1 & 1 & 1 & 1 & 1 & 1 & 1 & & 1 & 1 & 1 & 1 \\
\hline$a_{3}$ & 1 & & 1 & & & & & & & & & \\
\hline$a_{4}$ & 1 & & & & & & & & & & & \\
\hline$a_{5}$ & 1 & 1 & & & & & & & & & & \\
\hline$a_{6}$ & & & & 1 & & 1 & & & 1 & & 1 & \\
\hline$a_{7}$ & & & & 1 & 1 & 1 & & & 1 & 1 & 1 & \\
\hline$a_{8}$ & & & & 1 & 1 & & 1 & & 1 & 1 & & 1 \\
\hline$a_{9}$ & & & & & & & & 1 & & & & \\
\hline
\end{tabular}

Notice the common patterns in condition rows in $c_{6}$ and $c_{7}$, and action rows in $a_{6}, a_{7}$, and $a_{8}$.

Above in Example 2.1 is a flattened TDT. The first three columns have identical condition entries in $c_{1}$ and $c_{2}$, and identical action entries in $a_{1}$ and $a_{2}$. These columns differ in rows corresponding to conditions $\left\{c_{4}\right.$, $\left.c_{5}\right\}$ and actions $\left\{a_{3}, a_{4}, a_{5}\right\}$, which appear only in the first three columns. This may result, for example, from merging a sub-TDT consisting of only conditions $\left\{c_{4}\right.$, $\left.c_{5}\right\}$ and actions $\left\{a_{3}, a_{4}, a_{5}\right\}$.

Figure 1 shows a hierarchy of TDTs which specify the same behavior as Example 2.1. The equivalence can be verified by merging the hierarchy of TDTs [4]. Note that the conditions and actions are partitioned among these TDTs.

$T D T_{1}$ :
\begin{tabular}{|c||c|c|c|c|}
\hline$c_{1}$ & $\mathrm{Y}$ & $\mathrm{Y}$ & $\mathrm{Y}$ & $\mathrm{N}$ \\
\hline$c_{2}$ & $\mathrm{Y}$ & $\mathrm{N}$ & $\mathrm{N}$ & \\
\hline$c_{3}$ & & $\mathrm{Y}$ & $\mathrm{N}$ & \\
\hline \hline$a_{1}$ & 1 & & 1 & 1 \\
\hline$a_{2}$ & 1 & 1 & & 1 \\
\hline$T D T_{2}$ & 1 & & & \\
\hline$T D T_{3}$ & & 1 & & 1 \\
\hline$a_{9}$ & & & 1 & \\
\hline
\end{tabular}

\begin{tabular}{|c|c|c|c|c|}
\hline \multicolumn{4}{|c|}{$T D T_{2}:$} & \\
\hline$c_{4}$ & $\mathrm{Y}$ & $\mathrm{N}$ & $\mathrm{N}$ & \\
\hline$c_{5}$ & & $\bar{Y}$ & $\overline{\mathrm{N}}$ & \\
\hline$a_{3}$ & 7 & & 1 & \\
\hline$a_{4}$ & 1 & & & \\
\hline$a_{5}$ & 1 & 1 & & \\
\hline \multicolumn{5}{|c|}{$T D T_{3}:$} \\
\hline$c_{6}$ & $\bar{Y}$ & $\bar{Y}$ & $\mathrm{~N}$ & $\mathrm{~N}$ \\
\hline$c_{7}$ & $\bar{Y}$ & $\mathrm{~N}$ & $\bar{Y}$ & $\mathrm{~N}$ \\
\hline$\overline{\bar{a} a_{6}}$ & $\bar{~} 1$ & & $\overline{1}$ & \\
\hline$a_{7}$ & 1 & 1 & 1 & \\
\hline$a_{8}$ & 1 & 1 & & 1 \\
\hline
\end{tabular}

Figure 1: One possible decomposition of the TDT in Example 2.1.

The commonality in the flattened TDT may not result from multiple calls to a procedure as indicated by $T D T_{3}$ in Figure 1. It could also be a result of commonality in the input HDL specification. If this is the case, extraction will lead to a size reduction in the synthesized circuit.

It is not always possible to decompose a given TDT into a hierarchical TDT as shown in Figure 1 above. Neither is it always valid to merge the TDT hierarchy into flattened TDT [4]. These two transformations are valid only when the specified concurrency types, data dependencies, and serializations are preserved. In this particular example, we assume that the order of execution of all actions follows the order in which they appear in the condition stub. For the transformations to be valid, in this particular example, we also note that the actions $a_{1}$ and $a_{2}$ do not modify conditions in called TDTs namely $c_{4}$ through $c_{7}$.

The structural requirements for TDT decomposition can be efficiently captured by a two-level algebraic representation of TDTs [2]. This representation only captures the control dependencies in action sets and hence is strictly a sub set of TDT information. We also restrict the TDT model to only limited-entry forms, one where actions matrix is a Boolean matrix. The utility of this form has been demonstrated earlier with respect to modeling of exception conditions [4]. For each con- 
dition variable $c$, we define a positive condition literal, denoted as $l_{c}$, which corresponds to an ' $\mathrm{Y}$ ' value in a condition entry. We also define a negative condition literal, denoted as $l_{\bar{c}}$, which corresponds to an ' $\mathrm{N}$ ' value in a condition entry.

We define a '.' operator between two action literals and two conditions literals which represents a conjunction operation. This operation is both commutative and associative [4].

A TDT is a set of rules, each of which consists of a condition part which determines when the rule is selected, and an action part which lists the actions to be executed once a rule is selected for execution. The condition part of a rule is represented as

$$
\begin{gathered}
\prod_{c e(i) \neq{ }^{\prime} X^{\prime} ; i=1, \text { ncond }} \kappa_{i} \\
\kappa_{i}=\left\{\begin{array}{c}
l_{c_{i}}, \text { when } c e(i)=' \mathrm{Y} \\
l_{\bar{c}_{i}}, \text { when } c e(i)=' \mathrm{~N}
\end{array}\right.
\end{gathered}
$$

where ncond is the number of conditions in the TDT and $c e(i)$ is the condition entry value at the $i$ th condition row for this rule. The action part of a rule is represented as

$$
\prod_{a e(i) \neq 0^{\prime} 0^{\prime} ; i=1, \text { nact }} l_{a_{i}}
$$

where nact is the number of actions in the TDT and $a e(i)$ is the action entry value at the $i$ th action row for this rule. A rule is a tuple, denoted by

$$
(\mathcal{K}: \alpha)
$$

As will become clear later, for the purpose of TDT decomposition a rule can be expressed as a product of corresponding action and condition literals. We call such a product a cube. For a given TDT, $T$, we define an algebraic expression, $E_{T}$, that consists of disjunction of cubes corresponding to rules in $T$.

For simplicity, we can drop the '.' operator and ':' denotation and use ' $c$ ' or ' $a$ ' instead of $l_{c}$ and $l_{a}$ in the algebraic expressions of TDTs. These symbols follow only algebraic laws for symbolic computation. For treatment of this algebra, the reader is referred to [4].

Example 2.2. Here is the algebraic expression for the TDT in Example 2.1.

$$
\begin{aligned}
E_{T D T 2.1} & \\
& =c_{1} c_{2} c_{4} a_{1} a_{2} a_{3} a_{4} a_{5}+c_{1} c_{2} \bar{c}_{4} c_{5} a_{1} a_{2} a_{5} \\
& +c_{1} c_{2} \bar{c}_{4} \bar{c}_{5} a_{1} a_{2} a_{3}+c_{1} \bar{c}_{2} c_{3} c_{6} c_{7} a_{2} a_{6} a_{7} a_{8} \\
& +c_{1} \bar{c}_{2} c_{3} c_{6} \bar{c}_{7} a_{2} a_{7} a_{8}+c_{1} \bar{c}_{2} c_{3} \bar{c}_{6} c_{7} a_{2} a_{6} a_{7} \\
& +c_{1} \bar{c}_{2} c_{3} \bar{c}_{6} \bar{c}_{7} a_{2} a_{8}+c_{1} \bar{c}_{2} \bar{c}_{3} a_{1} a_{9} \\
& +\bar{c}_{1} c_{6} c_{7} a_{1} a_{2} a_{6} a_{7} a_{8}+\bar{c}_{1} c_{6} \bar{c}_{7} a_{1} a_{2} a_{7} a_{8} \\
& +\bar{c}_{1} \bar{c}_{6} c_{7} a_{1} a_{2} a_{6} a_{7}+\bar{c}_{1} \bar{c}_{6} \bar{c}_{7} a_{1} a_{2} a_{8}
\end{aligned}
$$

Note that there is no specification on delay, concurrency type, serialization relation, and data dependency. Also notice that ' $c$ ', ' $\bar{c}$ ', and ' $a$ ' are short-hand notations for ' $l_{c}$ ', ' $l_{\bar{c}}$ ', ' $l_{a}$ ' respectively.

\subsection{Kernel Extraction}

During TDT decomposition, it is important to keep an action literal or condition literal within one sub-TDT, that is, the decomposed TDTs must partition the condition and action literals. To capture this, we introduce the notion of TDT support.

Definition 2.1 The TDT-support of an expression $E_{T}$ is the set of action literals in $E_{T}$ and positive condition literals corresponding to condition literals that appear in $E_{T}$.

Example 2.3. Expression $c_{1} \bar{c}_{2} c_{3} \bar{c}_{6} \bar{c}_{7} a_{2} a_{8}$ is a cube. Its TDT support is $\left\{c_{1}, c_{2}, c_{3}, c_{6}, c_{7}, a_{1}, a_{8}\right\}$.

We consider here TDT decompositions into subTDTs that have disjoint TDT-supports. TDT decomposition uses algebraic division of TDT-expressions to identify sub TDTs. We define the algebraic division as follows:

Definition 2.2 Let $\left\{f_{d v n d}, f_{d v s r}, f_{q u o t}, f_{\text {rem }}\right\}$ be algebraic expressions. We say that $f_{d v s r}$ is an algebraic divisor of $f_{d v s r}$ when we have $f_{d v n d}=f_{d v s r} \cdot f_{q u o t}+f_{r e m}$, the TDT-support of $f_{d v s r}$ and the TDT-support of $f_{q u o t}$ are disjoint, and $f_{d v s r} \cdot f_{\text {quot }}$ is non-empty.

An algebraic divisor is called a factor when the remainder is void. An expression is said to be cube free when it cannot be factored by a cube.

Definition 2.3 A kernel of an expression is a cubefree quotient of the expression divided by a cube, which is called the co-kernel of the expression.

Example 2.4. Rewrite the algebraic form of $T D T_{\text {Example } 2.1}$ as follows.

$$
\begin{aligned}
& E_{T D T 2.1} \\
& =c_{1} c_{2} a_{1} a_{2}\left(c_{4} a_{3} a_{4} a_{5}+\bar{c}_{4} c_{5} a_{5}+\bar{c}_{4} \bar{c}_{5} a_{3}\right) \\
& +c_{1} \bar{c}_{2} c_{3} a_{2}\left(c_{6} c_{7} a_{6} a_{7} a_{8}+c_{6} \bar{c}_{7} a_{7} a_{8}+\bar{c}_{6} c_{7} a_{6} a_{7}+\bar{c}_{6} \bar{c}_{7} a_{8}\right) \\
& +c_{1} \bar{c}_{2} \bar{c}_{3} a_{1} a_{9} \\
& +\bar{c}_{1} a_{1} a_{2}\left(c_{6} c_{7} a_{6} a_{7} a_{8}+c_{6} \bar{c}_{7} a_{7} a_{8}+\bar{c}_{6} c_{7} a_{6} a_{7}+\bar{c}_{6} \bar{c}_{7} a_{8}\right)
\end{aligned}
$$

The expression $c_{4} a_{3} a_{4} a_{5}+\bar{c}_{4} c_{5} a_{5}+\bar{c}_{4} \bar{c}_{5} a_{3}$ is a kernel of $T D T_{\text {Example 2.1 }}$ with a corresponding co-kernel $c_{1} c_{2} a_{1} a_{2}$. Similarly, $c_{6} c_{7} a_{6} a_{7} a_{8}+c_{6} \bar{c}_{7} a_{7} a_{8}+\bar{c}_{6} c_{7} a_{6} a_{7}+\bar{c}_{6} \bar{c}_{7} a_{8}$ is also a kernel of $T D T_{\text {Example 2.1, with two corresponding co- }}$ kernels: $c_{1} \bar{c}_{2} c_{3} a_{2}$ and $\bar{c}_{1} a_{1} a_{2}$.

\section{Algorithm for TDT Decompo- sition}

This section presents an algorithm for TDT decomposition. The core of the algorithm is similar to the techniques used in multi-level logic optimization. Therefore we first discuss how to compute algebraic kernels from TDT-expressions before we show the complete algorithm which calls the kernel computing core and addresses some important issues such as preserving datadependencies between actions through TDT decomposition. 


\subsection{Algorithms for Kernel Extraction}

A naive way to compute the kernels of an expression is to divide it by the cubes corresponding to the power set of its support set. The quotients that are not cube free are weeded out, and the others are saved in the kernel set [5]. This procedure can be improved in two ways: (1) by introducing a recursive procedure that exploits the property that a kernel of a kernel of an expression is also the kernel of this expression, (2) by reducing the search by exploiting the commutativity of the '.' operator. Algorithm 3.1 shows a method adapted from a kernel extraction algorithm due to Brayton and McMullen [6], which takes into account the above two properties to reduce computational complexity.

\section{Algorithm 3.1 A Recursive Procedure Used in Kernel Extraction}

INPUT: a TDT expression $e$, a recursion index $j$; OUTPUT: the set of kernels of TDT expression $e$; extractKernelR $(e, j)\{$

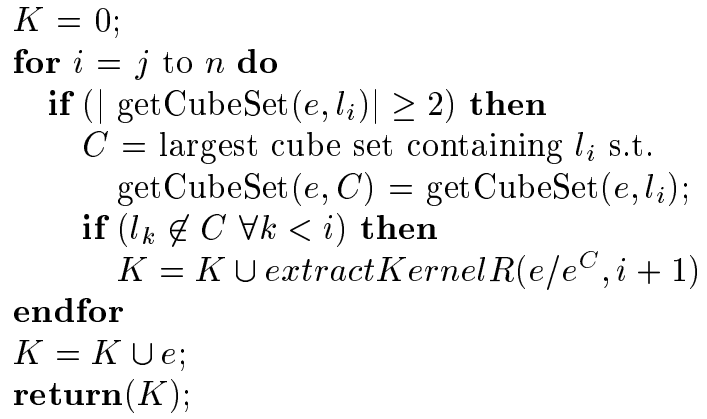

In the above algorithm, getCubeSet $(e, C)$ returns the set of cubes of $e$ whose support includes $C$. We order the literals so that condition literals appear before action literals. We use $n$ as the index of the last condition literal since a co-kernel containing only action literals does not correspond a valid TDT decomposition. Notice that $l_{c}$ and $l_{\bar{c}}$ are two different literals as we explained earlier. The algorithm is applicable to cube-free expressions. Thus, either the function $e$ is cube-free or it is made so by dividing it by its largest cube factor, determined by the intersection of the support sets of all its cubes.

Example 3.1. After running Algorithm 3.1 on the algebraic expression of $T D T_{2.1}$ we get the following kernels:

$$
\begin{aligned}
k_{1} & =E_{T D T 2.1} \\
k_{2} & =c_{2} a_{1} a_{2} c_{4} a_{3} a_{4} a_{5}+c_{2} a_{1} a_{2} \bar{c}_{4} c_{5} a_{5}+c_{2} a_{1} a_{2} \bar{c}_{4} \bar{c}_{5} a_{3} \\
& +\bar{c}_{2} c_{3} a_{2} c_{6} c_{7} a_{6} a_{7} a_{8}+\bar{c}_{2} c_{3} a_{2} c_{6} \bar{c}_{7} a_{7} a_{8}+\bar{c}_{2} c_{3} a_{2} \bar{c}_{6} c_{7} a_{6} a_{7} \\
& +\bar{c}_{2} c_{3} a_{2} \bar{c}_{6} \bar{c}_{7} a_{8} \\
k_{3} & =c_{4} a_{3} a_{4} a_{5}+\bar{c}_{4} c_{5} a_{5}+\bar{c}_{4} \bar{c}_{5} a_{3} \\
k_{4} & =c_{6} c_{7} a_{6} a_{7} a_{8}+c_{6} \bar{c}_{7} a_{7} a_{8}+\bar{c}_{6} c_{7} a_{6} a_{7}+\bar{c}_{6} \bar{c}_{7} a_{8} \\
k_{5} & =c_{5} a_{5}+\bar{c}_{5} a_{3} \\
k_{6} & =c_{7} a_{6}+\bar{c}_{7} \\
k_{7} & =c_{7} a_{6} a_{7}+\bar{c}_{7} a_{8}
\end{aligned}
$$

Note that $k_{6}$ has a cube with no action literals. This represents a TDT rule with no action selected for execution.

\subsection{TDT Decomposition}

Our TDT decomposition scheme works as follows. First, the algebraic expression of a TDT is constructed. Then a set of kernels are extracted from the algebraic expression. The kernels are eventually used to reconstruct a TDT representation in hierarchical form. Not all the algebraic kernels may be useful in TDT decomposition since the algebraic expression carries only a subset of the TDT information. We use a set of filtering procedures to delete from the kernel sets kernels which corresponds to invalid TDT transformations or transformations producing models that results in inferior synthesis results.

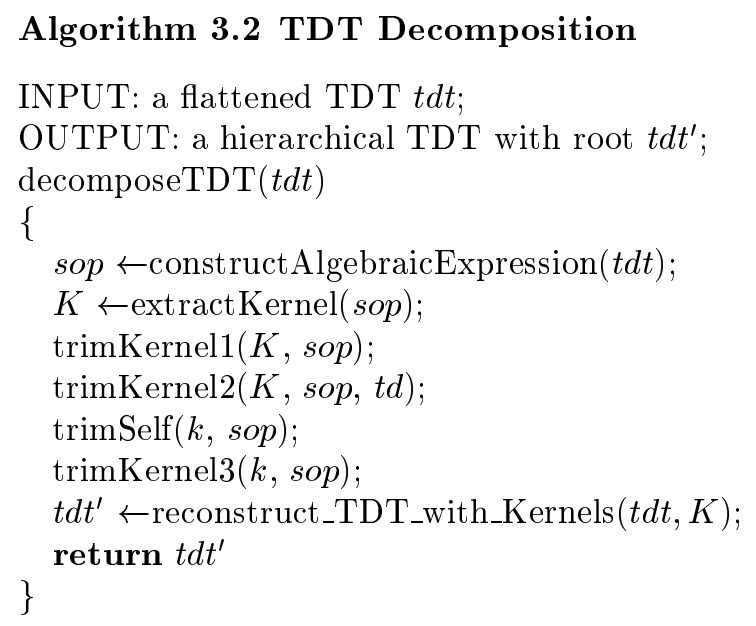

The procedure constructAlgebraicExpression() builds the algebraic expression of $t d t$ following Algorithm 3.3. The function expression() builds an expression out of a set of sets according to the data structure we choose for the two-level algebraic expression for TDTs. The complexity of the algorithm is $O(A R+C R)$ where $A$ is the number of action in $t d t, R$ is the number of rules in $t d t$, and $C$ is the number of conditions in $t d t$.

\section{Algorithm 3.3 Constructing Algebraic Expres- sions of TDTs}

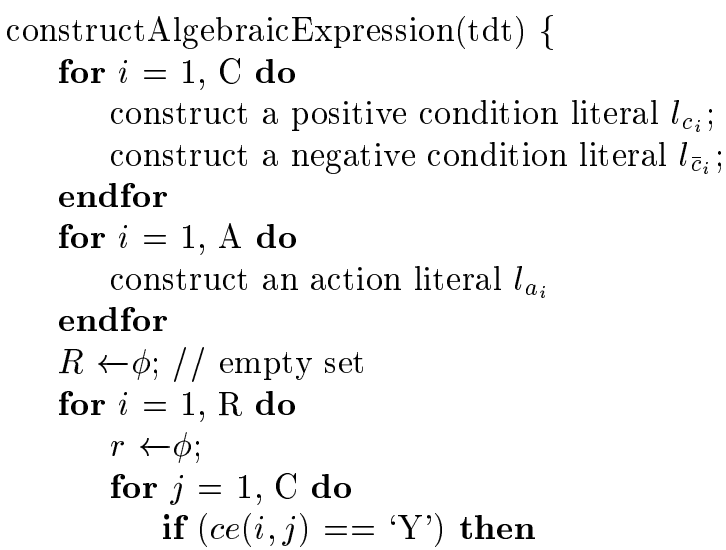




$$
\begin{gathered}
r \leftarrow r \cup\left\{l_{c_{i}}\right\} ; \\
\text { if }\left(c e(i, j)={ }^{\prime} \mathrm{N}^{\prime}\right) \text { then } \\
r \leftarrow r \cup\left\{l_{\bar{c}_{i}}\right\} ; \\
\text { endfor; } \\
R \leftarrow R \cup r ; \\
\text { endfor }
\end{gathered}
$$

\}

return expression $(R)$;

Procedure extractKernel(sop) calls the recursive procedure extractKernelR $(\operatorname{sop}, 1)$ to get a set of kernels of sop, the algebraic expression of $t d t$.

Some kernels appear only once in the algebraic expression of a TDT. These kernels would not help in reducing the resource requirement and therefore they are trimmed from $K$ using procedure trimKernel1(). The number of co-kernels corresponds to the number of times sub-TDT that corresponds to a certain kernel is called in the hierarchy of TDTs.

Since information such as data dependency are not captured in algebraic form of TDTs, the kernels in $K$ may not corresponds to a decomposition which preserves data-dependencies specified in the original TDT. These kernels are trimmed using procedure trimKernel2().

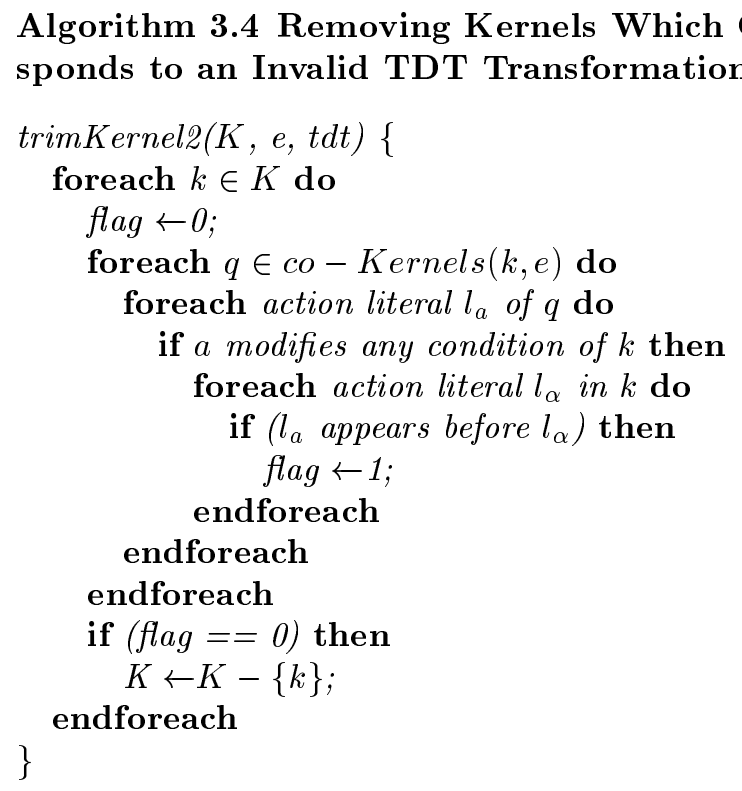

The worst case complexity of this algorithm is $O(A R+C R)$ since the program checks no more than once on each condition/action literal corresponding to a condition entry or action entry of $t d t$.

Example 3.2. Now we look at kernel $k_{4}=c_{6} c_{7} a_{6} a_{7} a_{8}+$ $c_{6} \bar{c}_{7} a_{7} a_{8}+\bar{c}_{6} c_{7} a_{6} a_{7}+\bar{c}_{6} \bar{c}_{7} a_{8}$. Suppose it corresponds to $T D T_{3}$. Also suppose in Example 2.1, $a_{2}$ modifies $c_{6}$ and the result of $a_{2}$ is also used in $a_{6}$. Because $a_{2}$ modifies $c_{6}$, in the hierarchical TDT we need to specify that $a_{2}$ comes after $T D T_{3}$ to preserve the behavior. However, this violates the data dependency specification between $a_{2}$ and $a_{6}$. Therefore, under this condition given above, $k_{4}$ will be eliminated by trimKernel2().

An expression may be a kernel of itself with a cokernel of ' 1 ' if it is kernel free. However this kernel is not useful for TDT decomposition. We use a procedure trimSelf() to delete an expression from its own kernel set for TDT decomposition. Also, as we mentioned earlier, a kernel of an expression's kernel is itself a kernel of this expression. However, in this paper, we consider TDT decomposition involving only two levels of calling hierarchies at a time. For this reason, we use trimKernel3() delete "smaller" kernels which are also kernels of other kernels of an expression.

Finally, we reconstruct a hierarchical TDT representation using the remaining algebraic kernels of the TDT expression. The algorithm is outlined below. It consists two procedures: reconstruct_TDT_with_Kernel(), and constructTDT() which is called by the other procedure to build a TDT out of an algebraic expression. Again, the worse case complexity of the algorithm is $O(C R+A R)$.

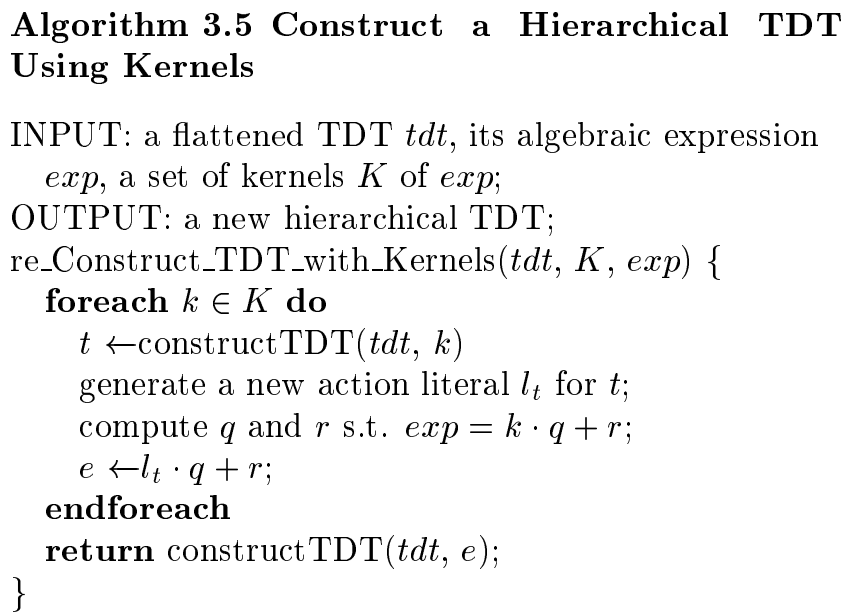

Algorithm 3.5 Construct a Hierarchical TDT Using Kernels

INPUT: a flattened TDT $t d t$, its algebraic expression exp, a set of kernels $K$ of exp;

OUTPUT: a new hierarchical TDT;

Example 3.3. Assume expression $c_{6} c_{7} a_{6} a_{7} a_{8}+c_{6} \bar{c}_{7} a_{7} a_{8}+$ $\bar{c}_{6} c_{7} a_{6} a_{7}+\bar{c}_{6} \bar{c}_{7} a_{8}$ is the only kernel left after trimming procedures performed on the kernel set of the algebraic expression of $T D T_{\text {Example 2.1 }}$. The following hierarchical TDT will be constructed.

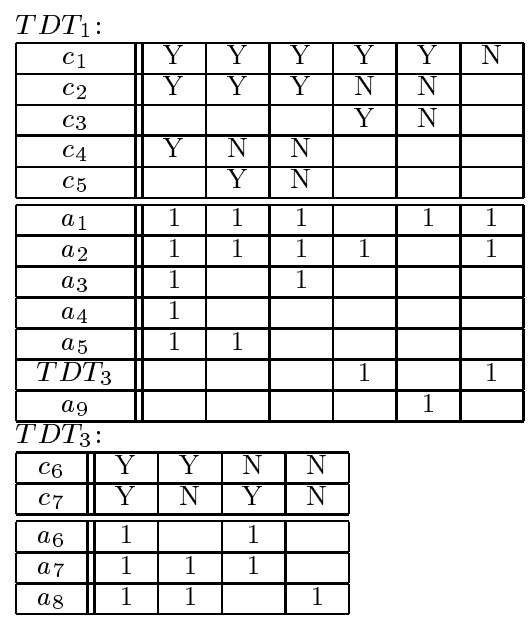




\section{Implementation and Experi- mental Results}

To show the effect of using TDT decomposition in presynthesis optimizations, we have incorporated our decomposition algorithm in PUMPKIN, the TDTbased presynthesis optimization tool [4]. Figure 2 shows the flow diagram of the process of presynthesis optimizations. The ellipse titled "kernel extraction" in Figure 2 shows where the TDT decomposition algorithm fits in the global picture of presynthesis optimizations using TDT.

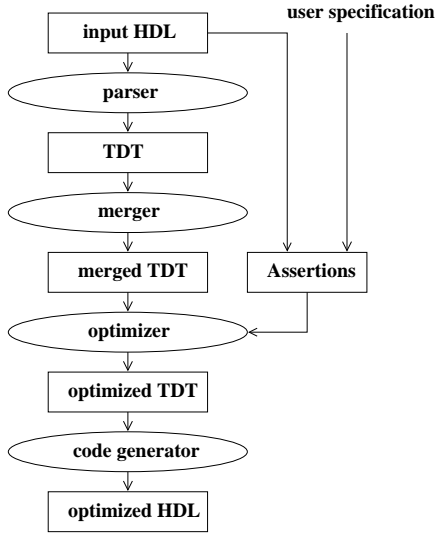

(a)

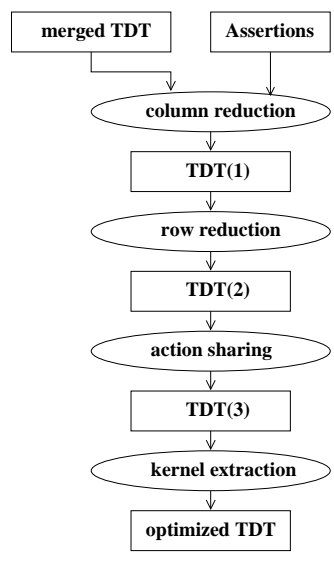

(b)
Figure 2: Flow diagram for presynthesis optimizations: (a) the whole picture, (b) details of the optimizer.

Our experimental methodology is as follows. The HDL description is compiled into TDT models, run through the optimizations, and finally output as a HardwareC description. This output is provided to the Olympus High-Level Synthesis System [7] for hardware synthesis under minimum area objectives. We use Olympus synthesis results to compare the effect of optimizations on hardware size. Hardware synthesis was performed for the target technology of LSI Logic 10K library of gates. Results are compared for final circuits sizes, in terms of number of cells. To evaluate the effectiveness of this step, we have turned off other optimizations and run PUMPKIN with several high-level synthesis benchmark designs.

Table 1 shows the results of TDT decomposition on examples designs. The design 'daio' refers to the HardwareC design of a Digital Audio Input-Output chip (DAIO) [8]. The design 'comm' refers to the HardwareC design of an Ethernet controller [9]. The design 'cruiser' refers to the HardwareC design of a vehicle controller. The description 'State' is the vehicle speed regulation module. All designs can be found in the highlevel synthesis benchmark suite [7]. The percentage of circuit size reduction is computed for each description and listed in the last column of Table 1 . Note that this improvement depends on the amount of commonality existing in the input behavioral descriptions.

Table 1: Synthesis Results: cell counts before and after TDT decomposition is carried out.

\begin{tabular}{|l|l|c|c|c|}
\hline design & \multirow{2}{*}{ module } & \multicolumn{2}{|c|}{ circuit size (cells) } & \multirow{2}{*}{$\%$} \\
\cline { 3 - 4 } & & before & after & \\
\hline \multirow{2}{*}{ daio } & phase_decoder & 1252 & 1232 & 2 \\
\cline { 2 - 4 } & receiver & 440 & 355 & 19 \\
\hline \multirow{2}{*}{ comm } & DMA_xmit & 992 & 770 & 22 \\
\cline { 2 - 4 } & exec_unit & 864 & 587 & 32 \\
\hline cruiser & State & 356 & 308 & 14 \\
\hline
\end{tabular}

\section{Conclusion and Future Work}

In this paper, we have introduced TDT decomposition as a complementary procedure to TDT merging. We have presented a TDT decomposition algorithm based on kernel extraction on an algebraic form of TDTs. Combining TDT decomposition and merging, we can restructure HDL descriptions to obtain descriptions that lead to either improved synthesis results or more efficient compiled code. Our experiment on named benchmarks shows a size reduction in the synthesized circuits after code restructuring.

As a future work, we are exploring HDL optimization strategies based various presynthesis optimization techniques that lead to best synthesis results.

Acknowledgment. This research is supported in part by NSF CAREER Award MIP 95-01615 and an FMC Education Fund Fellowship.

\section{References}

[1] J. Li and R. K. Gupta, "HDL Optimization Using Timed

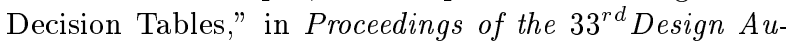
tomation Conference, pp. 51-54, June 1996.

[2] J. Li and R. K. Gupta, "Limited exception modeling and its use in presynthesis optimizations," in Proceedings of the $34^{\text {th }}$ Design Automation Conference, June 1997.

[3] D. D. Gajski, F. Vahid, S. Narayan, and J. Gong, Specification and Design of Embedded Systems. Prentice Hall, 1994.

[4] J. Li and R. K. Gupta, "System modeling and presynthesis using timed decision tables," Tech. Rep. UCI ICSTR-97-12, University of California, March 1997.

[5] G. D. Micheli, Synthesis and Optimization of Digital Circuits. McGraw-Hill, 1994.

[6] R. Brayton and C. McMullen, "The decomposition and factorization of boolean expressions," in Proceedings of the IEEE International Symposium on Circuits and Systems, 1982.

[7] G. D. Micheli, D. C. Ku, F. Mailhot, and T. Truong, "The Olympus Synthesis System for Digital Design," IEEE Design and Test Magazine, pp. 37-53, Oct. 1990.

[8] M. M. Ligthard, A. Bechtolsheim, G. D. Micheli, and A. E. Gamal, "Design of a digital input output chip," in Custom IC Conference, May 1989.

[9] D. Ku and G. D. Micheli, High-level Synthesis of ASICs under Timing and and Synchronization Constraints. Kluwer Academic Publishers, 1992. 